\title{
Influence of Triphenylphosphonium (TPP) Cation Hydrophobization with Phospholipids on Cellular Toxicity and Mitochondrial Selectivity
}

\author{
Diana Guzman-Villanueva*, Mark Ryan Mendiola, Huy Xuan Nguyen, Volkmar Weissig \\ Department of Pharmaceutical Sciences, Midwestern University College of Pharmacy-Glendale, Glendale, AZ 85308, USA
}

Received: December 17, 2014, Accepted: February 11, 2015, Published: February 19, 2015

*Corresponding author: Diana Guzman-Villanueva Ph.D., Postdoctoral Research Associate, Department of Pharmaceutical Sciences, College of Pharmacy-Glendale, Midwestern University, 19555 N. 59th Avenue, Glendale, AZ 85308, USA, Tel: +1-623-572-3597; E-mail: dguzma@midwestern.edu

\begin{abstract}
With the increase in the number of mitochondrial disruptionrelated pathologies (neurodegenerative diseases, cardiac injury, cancer, diabetes, etc.), and the lack of therapeutic approaches to address the dysfunctions rather than only treating the symptoms, the need for efficient therapeutic approaches is evident. Correcting these mitochondrial conditions, however, is still particularly challenging because the therapeutic alternatives are limited and requires specifically targeting the disease site. As a consequence of this, molecules that selectively accumulate within mitochondria represent the most feasible strategy. A common approach to target mitochondria is the use of lipophilic cations such as triphenylphosphonium (TPP), which is rapidly and extensively taken up by mitochondria due to their large membrane potential. However, TPP cations have also been associated to high cellular toxicity levels, which have limited their therapeutic use.
\end{abstract}

In order to reduce the cytotoxicity of the TPP cation, we synthesized a hydrophobized TPP moiety via a carboxyl-to-amine reaction using the two-tail phospholipid phosphatidylethanolamine (PE), which resulted in the CTPP-PE conjugate that was subsequently incorporated into liposomes at $5 \mathrm{~mol} \%$. After investigating the cytotoxicity and mitochondrial targeting activity of CTPP-PE liposomes under in vitro conditions, our results suggested that the CTPP-PE liposomes were less toxic than the previously reported STPP liposomes at concentrations as high as $300 \mu \mathrm{g} / \mathrm{mL}$, and that the functionality of the TPP cations was not altered by the hydrophobization reaction, as liposomes efficiently accumulated within mitochondria, as demonstrate by confocal microscopy. Thus, due to the low toxicity levels and high mitochondria selectivity shown by the CTPP-PE derivative, CTPP-PE liposomes represent an alternative to efficiently deliver mitochondrial-targeted drugs or active molecules in those disorders related to mitochondrial dysfunctions.

Keywords: CTPP-PE, Mitochondria, Liposomes, Cytotoxicity, Phosphatidylethanolamine, Targeting

\section{Introduction}

Mitochondria are well-defined subcellular organelles located in the cytosol of cells considered the main cell energy providers, genetic information storages, mediators of metabolic process such as oxidative phosphorylation, fatty acid oxidation, gluconeogenesis [1], as well as crucial active regulators of multiple cell death pathways [2]. Due to the large variety of biological process in which mitochondria are involved, it is not surprising that mitochondrial dysfunctions in the oxidative phosphorylation, redox balance (increased oxidative stress), or the introduction of mutations in the nuclear or mitochondrial DNA are implicated in a range of human diseases. Nevertheless, the variable number of clinical features shown by mitochondrial dysfunction makes them hard to identify since the symptoms are not specific or resemble to any mitochondrial disease in early phases and can vary from case to case [3].

Some of the clinical features associated to mitochondrial diseases may include neurological (epilepsy, dementia, speech disturbances, sensorineural deafness), cardiac (heart failure, cardiomyopathy), respiratory (respiratory failure, nocturnal hypoventilation, recurrent aspiration, apnoea), and endocrinal (diabetes, thyroid disease, parathyroid disease, ovarian failure) features [4]. Despite the difficulties in diagnosing mitochondrial disorders, there is enough evidence that suggests that mitochondrial dysfunction and oxidative stress play a key role in neurodegerative diseases such as Alzheimer and Parkinson diseases [5,6] and other pathologies including diabetes, cancer, and cardiovascular diseases [2].

Among those pathologies, it is well known that currently there are not therapies available to specifically treat mitochondrial dysfunctions but only to slow or treat the symptoms [7]. Nevertheless, since a large amount of these mitochondrial diseases are caused by an increase in reactive oxide species (ROS), dietary supplements and antioxidant therapy (vitamin E, CoQ10, L-carnitine, lipoic acid, riboflavina) has been thought as a feasible strategy to attenuate the excessive ROS production [8]. However, with the use of the antioxidant therapy to treat mitochondrial dysfunctions, high variability or not clinically relevant responses have been observed; as a consequence of their lack of ability to be efficiently internalized by the cells and selectively accumulate in the target organelle mitochondria [3]. 
To address this limitation several strategies or therapeutic approaches including mitochondrial-targeted delivery have been developed. One of the most useful strategies in targeting molecules to mitochondria consist of the conjugation of drugs and bioactive molecules to lipophilic cations such as triphenylphosphonium (TPP), because they can rapidly cross phospholipid bilayers without requiring a transporter and can be easily taken up into mitochondria [9]. Thus, the use of these lipophilic cations has led to an increase in the number of molecules (vitamin E, lipoic acid, etc) and delivery systems targeting mitochondria. In this regard, modifications of the TPP cation have allowed the development of mitochondrial-targeted delivery systems including liposomes. For example, Boddapati conjugated the TPP cation to the stearyl moiety and incorporated the modified STPP cation into liposomes. According to reported data these STPP liposomes efficiently accumulated into mitochondria and delivered drugs such as ceramide or anti-cancer drugs [10]. However, the non-specific toxicity of the TPP cations due to an excessive accumulation of the TPP cations within mitochondria still remained as the main drawback.

In this study, we modified the TPP cation via hydrophobization with the phospholipid phosphatidylethanolamine (PE) via a carboxyl-to-amine conjugation reaction, as a strategy to improve its cellular biocompatibility and to prepare TPP-PE liposomes with low toxicity levels as mitochondrial-targeted drug delivery systems. The effect of the modified TPP-PE and STPP liposomes on the cell viability, as well as their mitochondrial-targeting efficacy were evaluated under in vitro conditions using the cells line H9c2 and 4T1 after exposure to different liposomal concentrations.

\section{Methods}

\section{Materials}

The lipids L- $\alpha$-phosphatidylcoline (ePC, egg, chicken), cholesterol (chol, ovine wool), L- $\alpha$-phosphatidylethanolamine, 1,2-dioleoyl-sn-glycerol-3-phosphoethanolamine- $\mathrm{N}$-(lissamine rhodamine $B$ sulfonyl) (ammonium salt) (Rh-PE) were purchased from Avanti Polar Lipids Inc. (Alabaster, AL). N-(3Dimethylaminopropyl)- $N$-ethylcarbodiimide hydrochloride (EDCL) and $\mathrm{N}$-Hydroxysulfosuccinimide sodium salt (sulfoNHS) were purchased from Sigma-Aldrich (St. Louis, MO) (2-carboxyethyl)triphenylphosphanium chloride (CTPP) was purchased from Alfa Aesar (Ward Hill, MA). Stearyl triphenylphosphonium (STPP) was previously synthesized in our group. Spectra pore dialysis membrane was purchased from Spectrum Laboratories, Inc. (Rancho Dominguez, CA). DAPI Fluoromount-G was acquired from SouthernBiotech (Birmingham, AL), JC-1 mitochondrial membrane potential assay kit was purchased from Cayman Chemical Company (Ann Arbor, MI), CellTiter-Glo® Luminescent Cell Viability Assay was purchased from Promega (Madison, WI). Dulbecco's Modified Eagle's Medium (DMEM) and RPMI media were purchased from ATCC (Manassas, VA). Chloroform and methanol were used as provided.

\section{CTPP-PE synthesis and characterization}

The synthesis of the mitochondria-targeted CTPP-PE conjugate was carried out by a modified method, as previously described by Biswas [11] et al and Benein and co-workers [12]. Briefly, CTPP $(21.4 \mu \mathrm{mol})$ was dissolved in chloroform while stirring at room temperature, followed by the addition of EDCl $(64.5 \mu \mathrm{mol})$ and NHS $(64.5 \mu \mathrm{mol})$. After the mixture was stirred for approximately $2 \mathrm{~h}$, the phospholipid phosphatidylethanolamine (PE) $(16 \mu \mathrm{mol})$ was added. The mixture was incubated at room temperature for $20 \mathrm{~h}$, until the conjugation reaction took place. After the chloroform was evaporated, the mixture was resuspended in water and dialyzed for $24 \mathrm{~h}$, followed by the lyophilization of the product. Finally, the CTPP-PE obtained was dissolved in chloroform at $10 \mathrm{mg} / \mathrm{mL}$ concentration to be incorporated into liposomes.

\section{Liposome Preparation}

Multilamellar liposomes were prepared by the lipid film hydration method at a $10 \mathrm{mg} / \mathrm{mL}$ concentration. In brief, liposomes containing ePC and cholesterol (plain liposomes) or ePC, cholesterol and STPP (STPP liposomes), or CTPP-PE (CTPPPE liposomes) or PE (PE liposomes) were prepared at a 70:30 or 70:25:5 molar ratios, respectively (Table 1 ). The corresponding lipid mixtures in chloroform were dried on a rotary evaporator until obtaining a thin film. The lipid film was then hydrated with phosphate buffered saline (PBS), pH 7.4 and vortexed until the lipid was completely dispersed. To visualize the localization of the CTPP-PE liposomes within mitochondria, liposomes were labeled using $0.5 \mathrm{~mol} \% \mathrm{Rh}-\mathrm{PE}$.

\section{Liposome characterization}

After liposome preparation, the resulting liposomes were extruded through polycarbonate membranes of around $100 \mathrm{~nm}$ to reduce their size. The mean particle size, as well as the particle size distribution of the liposomes was determined by dynamic light scattering (DLS) using a Zetasizer Nano ZS Malvern system (Malvern Instrument Ltd., UK) after the liposomes were diluted in water. The zeta potential of the different liposome formulations was also determined after diluting the liposomal suspension in $1 \mathrm{mM} \mathrm{KCl}$ solution.

\section{Cell culture}

H9c2 (cardiomyocytes) and 4T1 (breast cancer) cells were cultured at $37^{\circ} \mathrm{C}$ with $5 \% \mathrm{CO}_{2}$, in Dulbecco's Modified Eagle Medium (DMEM) and RPMI-1640 medium, respectively, supplemented with $10 \%$ fetal bovine serum (FBS), $100 \mathrm{U} / \mathrm{mL}$ penicillin and $100 \mu \mathrm{g} / \mathrm{mL}$ streptomycin.

\section{In vitro compatibility of CTPP-PE liposomes}

In order to assess the biocompatibility of the CTPP-PE liposomes under in vitro conditions, approximately $2 \times 10^{4}$ H9c2 and 4T1 cells were seeded and incubated overnight. After washing the cells with PBS, the cells were treated with fresh media containing plain, STPP, CTPP-PE, or PE liposomes at 10 , 50,100 and $300 \mu \mathrm{g} / \mathrm{mL}$ for $24 \mathrm{~h}$ at $37^{\circ} \mathrm{C}$. After incubation, the number of viable cells was determined based on the ATP content. Luminescence measurements were recorded according to the manufacturer's instructions. The luminescence values obtained from the measurements were proportional to the ATP present in viable cells. 
Table 1: Description of the composition, particle size, polydispersity index (PDI), zeta potential of liposomal formulations.

\begin{tabular}{|c|c|c|c|}
\hline Lipid composition & Molar ratio & $\begin{array}{c}\text { Mean size } \\
\text { (nm) }\end{array}$ & PDI \\
\hline PC:Chol & $70: 30$ & $79 \pm 0.28$ & 0.2 \\
\hline PC:Chol:STPP & $70: 25: 5$ & $79 \pm 1.89$ & $-31 \pm 2.64$ \\
\hline PC:Chol:CTPP-PE & $70: 25: 5$ & $65 \pm 0.56$ & $-22 \pm 1.15$ \\
\hline PC:Chol:PE & $70: 25: 5$ & $85 \pm 1.145$ & $-11 \pm 0.41$ \\
\hline
\end{tabular}

\section{Mitochondrial membrane potential}

Mitochondrial membrane potential of H9c2 and 4T1 cells was evaluated after treatment with plain, STPP, CTPP-PE, and PE liposomes. Briefly, cells were exposed to liposomes using the concentrations $10,50,100$ and $300 \mu \mathrm{g} / \mathrm{mL}$ for $24 \mathrm{~h}$ at $37^{\circ} \mathrm{C}$, followed by the determination of mitochondria membrane potential using the JC-1 mitochondrial membrane potential assay. After the liposomal treatment, the cells were stained with the JC-1 solution and incubated for 15-30 min, according to the manufacturer's protocol. Healthy cells with high membrane potential were differentiated from unhealthy cells (green fluorescent monomers) by the formation of red fluorescent aggregates. The fluorescence intensity was determined with excitation and emission at 560 and $595 \mathrm{~nm}$, respectively, for aggregates, and $548 \mathrm{~nm}$ and $535 \mathrm{~nm}$ for monomers. The fluorescent intensity ratio of aggregates to monomers was used as an indicator of cell health.

\section{CTPP-PE liposomes targeting mitochondria}

H9c2 and 4T1 cells $\left(2 \times 10^{4}\right)$ were seeded into 24 -well plates containing coverslips and let attached to the plate overnight. Next day, the cells were treated with $50 \mu \mathrm{g} / \mathrm{mL} \mathrm{Rh}$-containing-plain or CTPP-PE liposomes and incubated for $24 \mathrm{~h}$ protected from light. After exposure with Rh-containing-CTPP-PE liposomes, the cells were washed three times with PBS solution. FBS-free media containing $200 \mathrm{nM}$ MitoTracker Green FM was added to the cells, followed by $30 \mathrm{~min}$ incubation at $37^{\circ} \mathrm{C}$ to stain mitochondria. After three washes with PBS solution, the coverslips were removed from the plate and mounted on microscope slides containing the DAPI Fluoromount-G nuclear probe. Mitochondria targeting of CTPP-PE liposomes was visualized using a Leica confocal microscope system using filters with an excitation light for DAPI of $455 \mathrm{~nm}, 490$ for MitoTracker Green, and $560 \mathrm{~nm}$ for Rhodamine at 40X magnification. The software Leica Application Suite, Advanced Fluorescence Lite 2.6 .3 build 8173 was used to analyze the acquired images. The corresponding fluorescent micrographs were merged to visualize Rh-containing plain and CTPP-PE liposomes localization within the cells. Mitochondria stained in yellow color were an indicator that $\mathrm{Rh}$-liposomes were taken up by the cells and reached mitochondria.

\section{Statistical analysis}

All results represent the mean \pm standard deviation of three independent experiments. Statistical significance was calculated using ANOVA test in GraphPad Prism 6.02 software.

\section{Results}

\section{CTPP-PE synthesis and characterization}

The hydrophobization of the TPP cation was carried out by using the phospholipid phosphatidylethanolamine via a carboxylto-amine conjugation reaction using the crosslinker EDCl and Sulfo-NHS, leading to the formation of the CTPP-PE conjugate (Figure 1). The conjugation reaction process was monitored by thin-layer chromatography (TLC). After approximately 20h, the product was dialyzed against distilled water for $24 \mathrm{~h}$ to remove the unreacted materials as previously reported [11]. Finally, the CTPP-PE derivative was obtained as a purified lyophilized powder that was dissolved in chloroform to be incorporated in the lipid mixture to prepare CTPP-PE liposomes. As previously characterized by Boddapati [10] and Biswas [11], typically the TPP cation is identified by the presence of signals of the protons corresponding to the aromatic groups at around 7.68 to $7.88 \mathrm{ppm}$ when characterized by ${ }^{1} \mathrm{H}-\mathrm{NMR}$, while the signals corresponding to the phosphorus group of the phospholipid and the TPP cation appear at 1 and $25.34 \mathrm{ppm}$, respectively, as characterized by ${ }^{31} \mathrm{P}-\mathrm{NMR}$.

\section{Preparation and liposome characterization}

Plain (PC:Chol), CTPP-PE (PC:Chol:CTPP-PE), STPP (PC:Chol:STPP) and PE (PC:Chol:PE) liposomes were prepared by the thin film hydration method and extruded though polycarbonate membranes to reduce their size. As determined by DLS analysis, the mean particle size for each liposomal formulation was around $80 \mathrm{~nm}$, as shown in Figure 2. In almost all cases, the PDI (polydispersity index) resulted in values $<0.2$, suggesting the presence of a homogenous size distribution, especially for the CTPP-PE liposomes that showed a monodisperse distribution with PDI values of 0.08 . Regarding the zeta potential, all the liposomal formulations showed negative values, ranging from -39 to -11 , being plain and PE liposomes the most negatively charged. As seen in Figure 2b, those liposomes that incorporated the TPP-PE derivative showed significantly less negative zeta potential values (Table 1), which agree with the presence of the TPP cation.

Liposomes with positive values have been only reached by using very high TPP concentrations (around 10-20 mol\%), as reported by Boddapati.

\section{In vitro compatibility of CTPP-PE liposomes}

In order to determine the biocompatibility of the CTPP-PE liposomes, H9c2 and 4T2 cells were treated for $24 \mathrm{~h}$ with different liposomal concentrations. Due to the ATP present in cells is an 
<smiles>O=C(O)CP(c1ccccc1)(c1ccccc1)(c1ccccc1)c1ccccc1</smiles>

Carboxyethyl-TPP (CTPP)

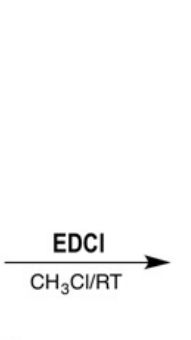

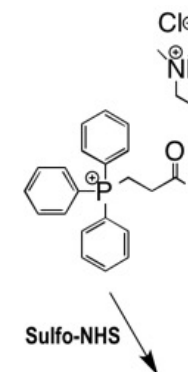

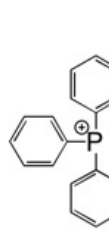

\section{le}<smiles>[AlH2]</smiles>

O-Acylisourea

(n)

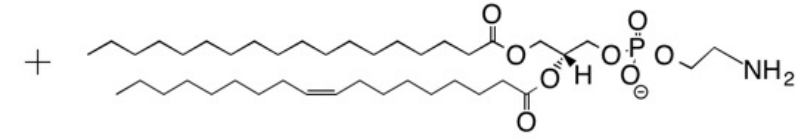

$\mathrm{O}_{\mathrm{O}}^{-1} \mathrm{O}^{\circ}$

L-alpha-phosphatidylethanolamine

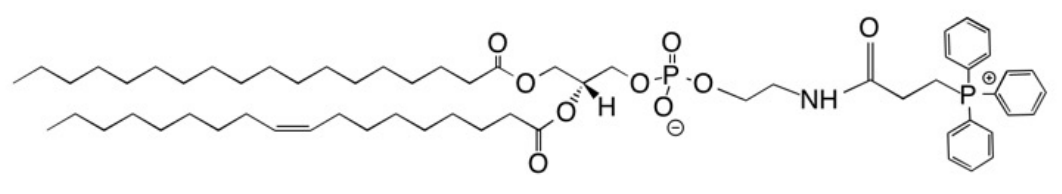

CTPP-PE Conjugate

Figure 1: Schematic representation of the CTPP-PE conjugate synthesis via carboxyl-to amino reaction.
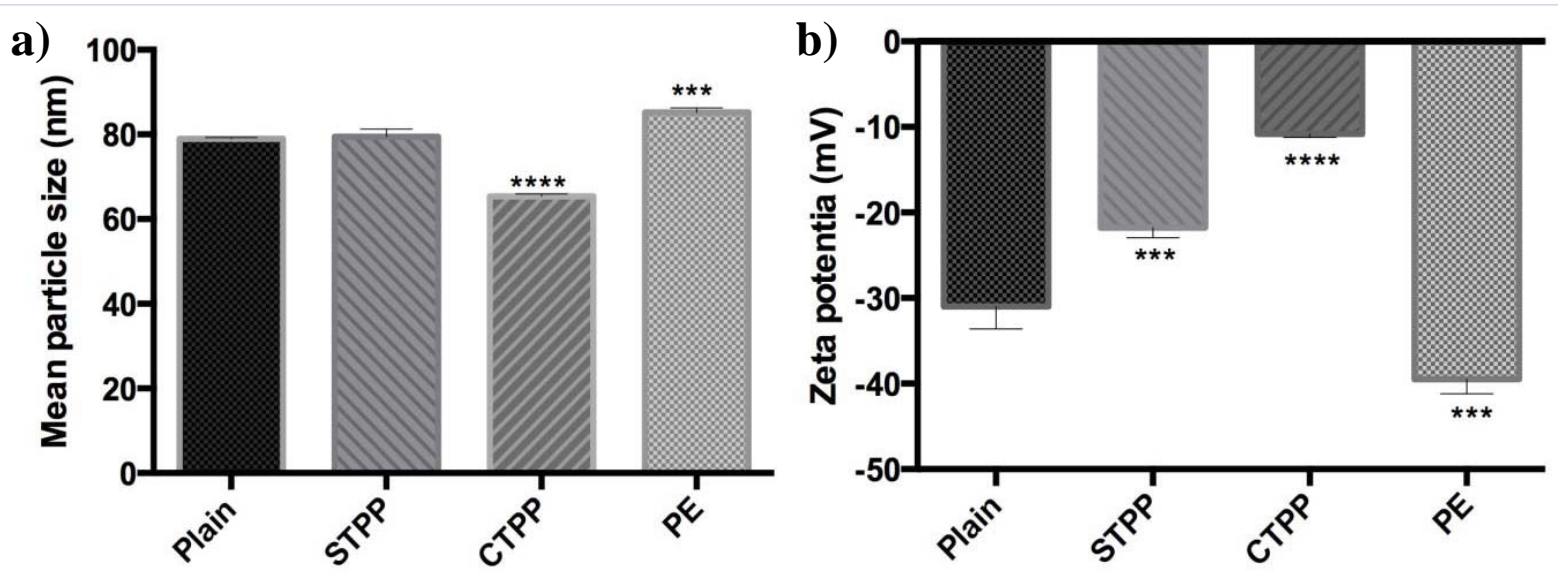

Figure 2: a) Particle size and b) zeta potential characterization of CTPP-PE liposomes. The particle size and zeta potential of modified liposomes containing $5 \mathrm{~mol} \%$ CTPP or STPP cations were evaluated after preparation. Data represent the mean \pm SD. $p<0.05$ values were statistically significant different.

indicator of metabolically active cells, when the cells are dying their ATP levels drop to the point where it is no longer possible for them to continue with their basic metabolic functions, which ultimately triggers their death. Therefore, in this assay the ATP content in cells treated with plain, STPP, CTPP-PE and PEliposomes was used to investigate their influence on cell viability after exposure.

As shown in Figure 3, STPP liposomes rapidly reduced the ATP levels in 4T1 cells, and therefore, cell viability to around 50\% when the lowest liposomal concentration was used $(10 \mu \mathrm{g} / \mathrm{mL})$, followed by complete cell depletion after the cells were treated with $300 \mu \mathrm{g} / \mathrm{mL}$ STPP liposomes, as seen in Figure 3. H9c2 cells also showed the same tendency as $4 \mathrm{~T} 1$ cells and drastically decreased the ATP levels when treated with STPP.

On the other hand, H9c2 cells treated with CTPP-PE maintained their cell viability as high as those cells treated with plain liposomes (91\%) after 24h (Figure 3a), even at the highest concentration $(300 \mu \mathrm{g} / \mathrm{mL})$. In contrast, the $4 \mathrm{~T} 1$ cell line showed higher sensitivity to the liposomal treatments reducing slightly more the cell viability at the highest concentration (Figure 3b), 


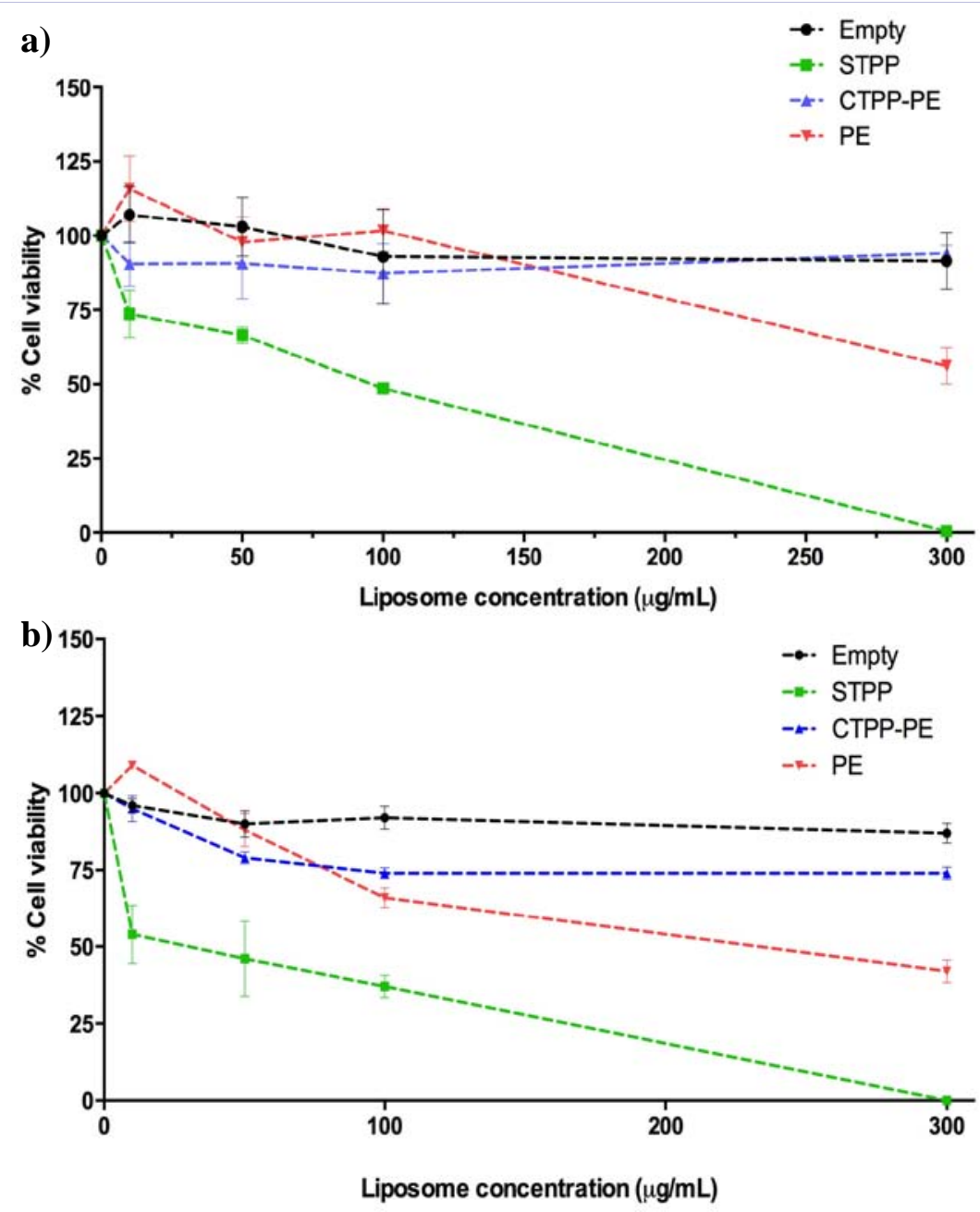

Figure 3: In vitro evaluation of the CTPP-PE liposomes. The effect of TPP-bearing liposomes on the (a) H9c2 and (b) 4T1 cell viability was determined after $24 \mathrm{~h}$ incubation with different liposomal concentrations.

phenomena that has also been reported in other breast cancer cell lines such as BT20 [10], suggesting that in comparison to non-transformed cells (normal), transformed cells (cancer cells), may be more sensible to increased concentration of TPP moiety.

The high \% cell viability observed during the treatment with CTPP-PEliposomes suggested that the conjugation of the lipophilic TPP cation to the phospholipid phosphatidylethanolamine (PE) significantly reduced its cytotoxicity when using up to $5 \mathrm{~mol} \%$ TPP. In contrast, STPP liposome showed to be highly toxic, as the cell viability was drastically reduced at low STPP liposome concentration, as previously reported in the literature.

The cell viability of $\mathrm{H} 9 \mathrm{c} 2$ and $4 \mathrm{~T} 1$ cells treated with $\mathrm{PE}$ liposomes remained intact at low concentrations. Nevertheless, it showed to be toxic at high concentrations, as a reduction in cell viability was observed.

\section{Mitochondrial membrane potential}

Mitochondrial disruption constitutes one of the early features of cell death. Among these disrupting effects, changes in mitochondrial membrane potential $(\Delta \Psi \mathrm{m})$ are included. It is well known that $\Delta \Psi \mathrm{m}$ is critical to maintain metabolic activity in cells, especially the ATP production; thus, the loss of $\Delta \Psi \mathrm{m}$ often results in damage of the respiratory chain, which eventually reduces the energy levels in cells triggering their death. In order to evaluate any possible alteration in mitochondrial function or depletion of mitochondrial membrane potential due to plain CTPP-PE, STPP and PE liposomal exposure, H9c2 and 4T1 cells were evaluated using the mitochondrial membrane potential assay JC-1. JC-1 is a lipophilic cationic dye able to detect mitochondrial depolarization. When the cells suffer mitochondrial depolarization a decreased in the red/green fluorescence intensity ratio is observed during the assay.

As illustrated in Figure 4, the aggregate (red, healthy)/ monomer (green, unhealthy) ratio was very low in all concentrations when the cells were treated with STPPcontaining liposomes, due to its well-documented cytotoxicity. In contrast, cells treated with CTPP-PE liposomes showed a high mitochondrial membrane potential as those cells treated with plain liposomes and untreated cells (data not shown). Thus, these 


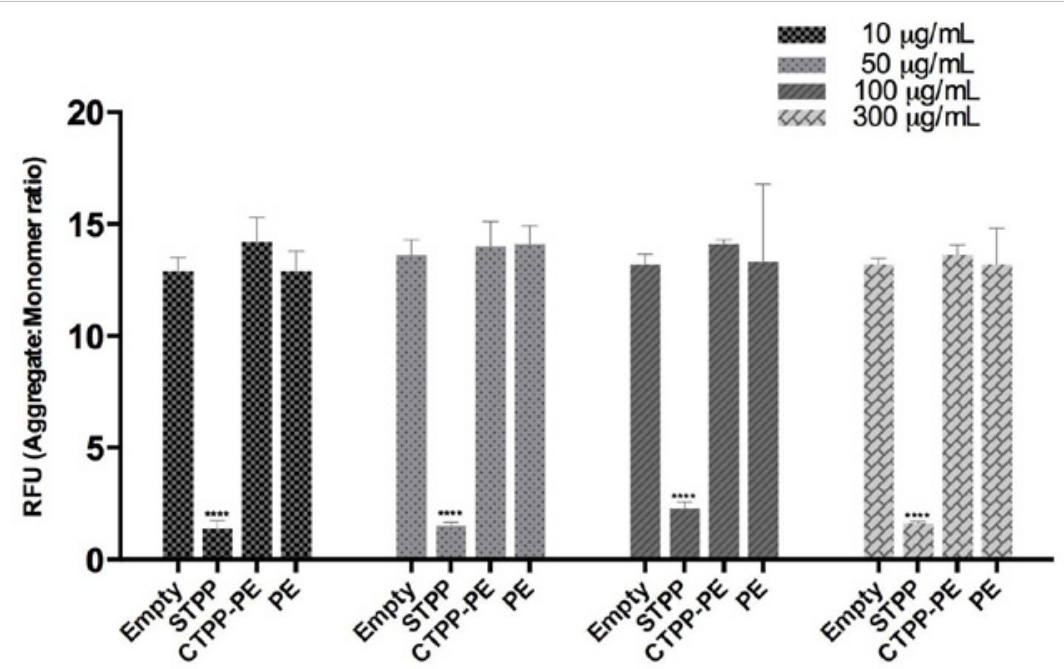

Figure 4: JC-1 mitochondrial membrane potential determination assay. The mitochondria membrane potential was determined using the JC-1 assay after $\mathrm{H} 9 \mathrm{c} 2$ cells were treated with mitochondria-targeted liposomes at 10, 50, 100 and $300 \mu \mathrm{g} / \mathrm{mL}$ for $24 \mathrm{~h}$. p $<0.05$ values were statistically significant different.
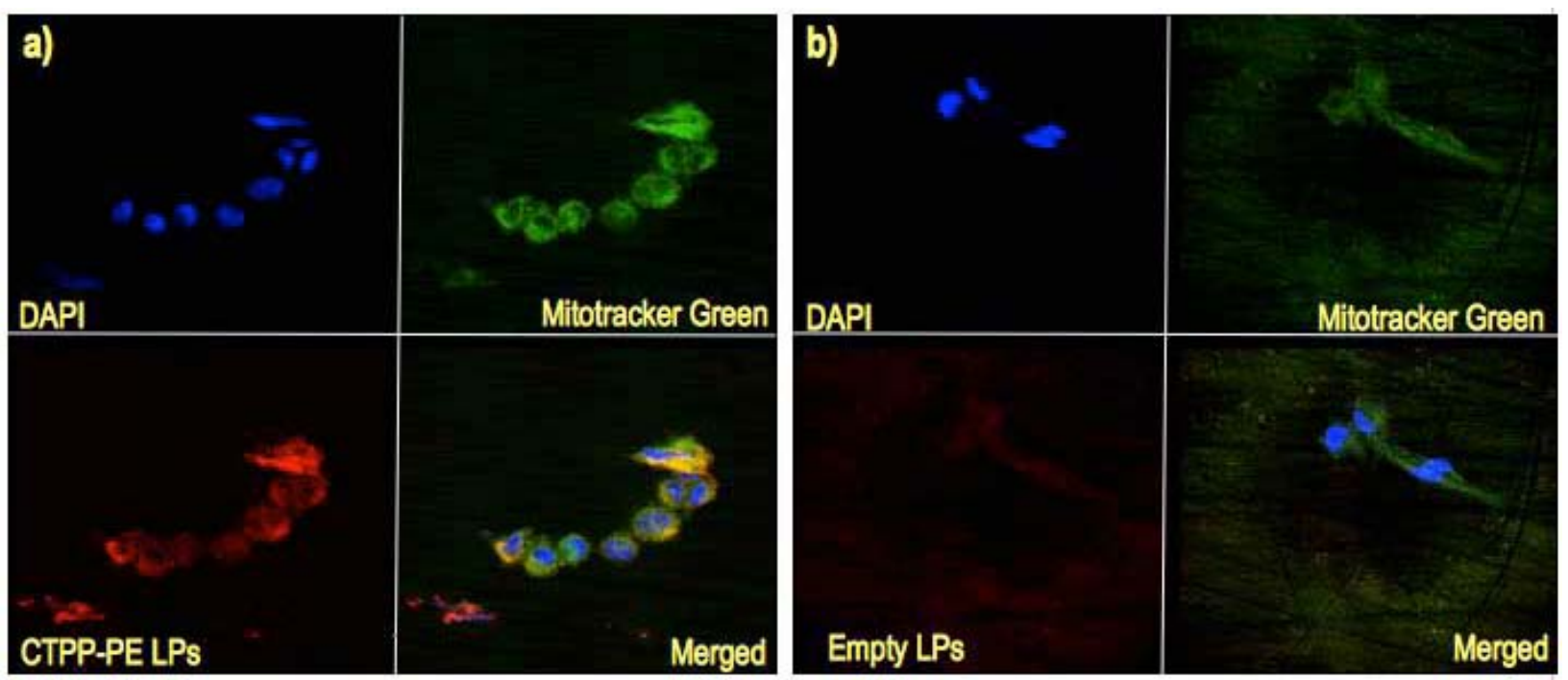

Figure 5: Cellular uptake and mitochondrial-targeting characterization of CTPP-PE liposomes by confocal microscopy. H9c2 cells were treated with: a) the modified liposomes CTPP-PE ( $50 \mu \mathrm{g} / \mathrm{mL}$ ) or b) empty liposomes at the same concentration for 24h. CTPP-PE and empty liposomes were labeled with Rhodamine (Rh, in red). Mitochondria were stained with the Mitotracker Green probe, while nuclei were stained with DAPI. Liposomes were located within mitochondria as shown by the yellow color observed after the micrographs were overlaid.

results confirmed that CTPP-PE liposomes show low toxicity effects at concentrations up to $5 \mathrm{~mol} \%$, when evaluated under in vitro conditions as no changes in the mitochondrial membrane potential were seen up to $24 \mathrm{~h}$.

\section{CTPP-PE liposomes uptake and mitochondria targeting}

In order to evaluate the cellular uptake of the CTPP-PE liposomes and determine any possible TPP targeting activity modification due to the carboxyl-to-amine conjugation reaction with PE, H9c2 and 4T1 cells were treated with CTPP-PE. To visualize the liposome location in the cell the liposomes were labeled with Rhodamine at $0.5 \mathrm{~mol} \%$, while the mitochondrial probe MitoTracker Green was used to stain mitochondria. As previously mentioned in the methods section, the presence of yellow dots, as a result of the green and red fluorescent filters overlaying in the confocal micrographs, was an indicator that CTPP-PE liposomes were located within mitochondria. As shown in Figure 5, the CTPP-PE liposomes were not only highly internalized by the cells but they were confined within mitochondria as a high yellow intensity was observed in the overlaid micrographs. As a comparison of not targeting activity, the cells were also treated with plain liposomes (PC:Chol:Rh), and as expected, little or none mitochondrial activity was observed.

\section{Discussion}

It has been well described in the literature the crucial 
role of mitochondria in metabolic activities such as oxidative phosphorylation, as well as in other important activities including apoptotic and necrotic pathways. Due to their functionality and participation in these multiple roles, any mitochondrial dysfunction contributes to a series of events that generate a wide range of pathologies $[13,14]$. Among these mitochondrial disorders Alzheimer, ischemia-reperfusion injury, inflammation, and cancer are well known; however, chronic progressive external ophthalmoplegia (CPEO), mitochondrial encephalomyopathy with lactic acidosis and stroke-like episodes (MELAS), or Pearson syndrome are other 'rare' diseases associate to mitochondrial abnormalities that are poorly treated $[13,15]$.

In practice, treatments for mitochondrial diseases are not therapeutically successful yet. Therefore, the management of some of these mitochondrial disorders includes only symptombased treatment or preventing measures to avoid symptom worsening such as the oral administration of riboflavin and coenzyme Q10 for patients with complex I and/ or complex II deficiency or ubiquinone deficiency, respectively [16]. However, high concentrations of these drugs are required to reach an effective concentration at the disease site, which as a consequence could result in a variety of toxic side effects [14]. In this regard, the idea of mitochondria targeting as a therapeutic strategy was proposed to treat those diseases with mitochondrial dysfunctions. Thus, a number of methods have been developed to selectively target mitochondria; however, due to their unique characteristics mitochondrial drug therapy still represents a challenge [17].

To date, lipophilic cations represent the most commonly used method to target mitochondria. Among these, triphenylphosphonium (TPP) is a lipophilic cation characterized by a large hydrophobic surface area, which allows it to permeate throughout phospholipid bilayers and accumulates within mitochondria [18]. Nevertheless, it has been documented that its excessive accumulation in mitochondria is able to disrupt membrane integrity and alters metabolic functions such as respiratory chain and ATP synthesis $[18,19]$.

In one of the multiple attempts to deliver drugs to mitochondria, the idea of using mitochondria-specific liposomes as drug carriers was raised by Boddapati and Weissig [10], who worked in the hydrophobization of the TPP cation using stearyl bromide to facilitate their incorporation into liposomal bilayers, resulting in the mitochondria targeting stearyl triphenylphosphonium (STPP) bromide, which was used to prepare STPP liposomes. The data obtained by Boddapati showed that the modification of the TPP cations to STPP cations and their incorporation into liposomes did not modify the ability of TPP to accumulates into mitochondria and thus, biological molecules, drugs like paclitaxel and antioxidants like vitamin E, were readily incorporated into the liposomes. However, the incorporation of STPP cations into liposomes at ratios ranging from 1 to $20 \mathrm{~mol} \%$ was still highly toxic for the cells $[10,11]$.

Our work, as a contribution to sub-cellular targeting, was aimed to synthesize a TPP moiety and prepare liposomes targeting mitochondria with reduced cell toxicity. Different to the conjugation of TPP to the block DSPE-PEG-2000amine, as synthesized by Tochilin [11] or to the distal end of the hexanolamine residue as described by D'Souza [12], our hydrophobized TPP cations consisted on the direct conjugation of the TPP cation to the amino group of the two-tail phospholipid phosphatidylethanolamine via carboxyl-to-amine reaction using the carbodiimide crosslinker EDCl and Sulfo-NHS. The modified TPP-PE moiety was then incorporated on the surface of several liposomal formulations to be evaluated under in vitro conditions.

As it is expected the toxicity of TPP cations also occurs under in vivo conditions [18], this may represent its major limiting factor for clinical applications. Thus, by the hydrophobization of TPP cations to CTPP-PE using phosphatidylethanolamine, instead the stearyl residue STPP, we significantly reduced of the cytotoxicity of TPP when incorporated into the liposomes. At the same concentration $(300 \mu \mathrm{g} / \mathrm{mL})$ and mol \% ratio (5) in $\mathrm{H} 9 \mathrm{c} 2$ and 4T1 cells, for example, STPP liposomes completely killed the cells, while CTPP-PE liposomes were able to maintain the cell viability at high levels in both cell lines up to $24 \mathrm{~h}$.

The observed high cytotoxicity of STPP may be related to its high similarity to alkyl-lysolipid-like structures. Lysolipids (single-tailed lipids) are non-bilayer forming lipids that tend to accumulate in the intracellular membrane generating high positive curvatures and membrane arrangements that contribute to the permeabilization of the mitochondria membrane by creating instability and modifying the properties of the lipid bilayer [20]. Along with these membrane structural modifications, we suggest that STPP may induce cell death by disturbing the lipid metabolism and lipid signaling in cell membranes, as described by van del Luit et al [21] and Marco and co-workers [22]. In addition, it has been described that the excessive accumulation of lysolipids in membranes induces cell lysis [23], which is consistent with concentration-dependent toxicity observed in the cells treated with STPP, as shown in Figure 3.

Regarding the mitochondrial membrane potential, Van Zutphen and Van Deenen [24], Lee and Chan [25] reported that the membrane electrical resistance is lowered while the permeability is increased when lysolipids are added to the cells as a consequence of the stress or tension introduced to the membrane [23]. For this reason, it is not surprising to see a decrease in the mitochondrial membrane potential of H9c2 and 4T1 cells when we treated with STPP liposomes.

In contrast to STPP (single-tailed), we suggest that the reduced cytotoxicity shown by the CTPP-PE could be highly related to the presence of the two long fatty acid hydrocarbon chains in the conjugate, which seems to allow a better integration of the CTPP-PE residue into the membrane bilayer with other lipids in a more symmetrical distribution than STPP, which mismatches the components of the lipid bilayer. Thus, by the reducing the high curvatures formed by STPP, CTPP-PE avoided the reorganization of the membrane lipids between the bilayers, bilayer instability or disruption, reduction of mitochondrial membrane potential, and membrane permeabilization. Although it has been described 
that the inclusion of PE also imposes a curvature stress in the membrane, as an inherent function to stabilize membrane proteins to their optimal conformations and modulate their activities, it has been reported that these curvatures are very small. Therefore, we presume that by using two-tail lipids like PE the curvature stress in the membranes caused by STPP will be alleviated and will avoid or reduce the membrane "reshape" that could lead to membrane disruption and alteration of the lipid metabolism. However, in order to clarify the mechanism associated to the reduction of the cytotoxicity by the CTPP-PE conjugate and study the behavior of other two-tail lipids further investigation is required.

As above described, two of the most important features that make the lipophilic TPP cations effective in delivering drugs to mitochondria are: 1) that they can easily pass though cellular membranes, and 2) that they accumulate within mitochondria due to the large mitochondrial membrane potential. Based on these properties, we prepared mitochondria-targeting CTPP$\mathrm{PE}$ liposomes and compared their cellular internalization and mitochondrial specificity to the conventional plain liposomes using fluorescence labeled liposomes. By confocal microscopy we showed that CTPP-PE liposomes were internalized by the cell and efficiently reached mitochondria as confirmed by the intense yellow color shown in the micrographs.

The underlying mechanisms by which mitochondriotropic low-molecular weight molecules target mitochondria have been exhaustively discussed earlier by Horobin et al [26] In brief, the main mechanism by which the CTPP-PE conjugates selectively target mitochondria is attributed to the hydrophobic surface and the positive delocalized charge of the TPP cation, which facilitate its movement through the phospholipid bilayers without requiring a transporter, followed by its accumulation into mitochondria driven by the large mitochondrial membrane potential (150-180 $\mathrm{mV}$, negatively inside). In addition, we presume that the hydrophobicity of the two long fatty acid hydrocarbon chains in CTPP-PE may contribute to lowering its activation energy to pass through the cells membrane, thus facilitating its uptake [19].

In summary, after we conjugated the TPP cation to the amino group present in the phospholipid phosphatidylethanolamine, we incorporated the CTPP-PE derivative into the liposomal bilayers and investigated their effect on two different cells lines, H9c2 and 4T1. From the data obtained, we could conclude that in addition to the significant reduction of the cytotoxicity of the TPP cation, its functionality was not affected by the direct hydrophobization reaction with PE. Together these results are consistent with the data previously reported by Torchilin and D'Souza $[11,12]$. Although Torchilin and D'Souza introduced larger or more hydrophobic residues to the TPP cation no difference in the behavior of TPP was observed when we directly attached the amino group in phospholipid $\mathrm{PE}$, which might suggest that the length or the hydrophobicity of the chain attached to the TPP cations does not alter the natural interaction of the TPP head groups with the membrane, such that its specificity for mitochondrial accumulation was not affected. These data is supported by Finichiu [9] and Ross [27], who reported that mitochondria uptake occurred using a variety of TPP moieties ranging from the simply TPP cation to TPP conjugated with alkyl groups with 1 to 5 carbons in length, carboxylic acids (10-carboxydecyl-TPP) and amines (10-aminodecyl-TPP), when using an isolated mitochondria model.

The hydrophobization of the TPP cation with the two tail phospholipid phosphatidylethanolamine, CTPP-PE, and its incorporation into liposomes significantly reduced the toxicity associated to the TPP cation without affecting its inherent ability to accumulate within mitochondria, thus, enabling the development of non-toxic carriers to efficiently deliver drugs or other active molecules to those cells with mitochondrial dysfunctions.

\section{Acknowledgments}

The authors would like to thank Midwestern University, College of Pharmacy-Glendale for the funding provided to support this work.

\section{References}

1. Gvozdjáková A. Mitochondrial Physiology. In: Gvozdjáková A, editor. Mitochondrial Medicine. Netherlands: Springer; 2008. p. 1-2.

2. Ubah OC, Wallace HM. Cancer therapy: Targeting mitochondria and other sub-cellular organelles. Curr Pharm Des. 2014; 20(2): 201-222.

3. Pieczenik SR, Neustadt J. Mitochondrial dysfunction and molecular pathways of disease. Exp Mol Pathol. 2007; 83(1): 84-92.

4. Taylor RW, Turnbull DM. Mitochondrial DNA mutations in human disease. 2005. Nat Rev Genet. 2005; 6(5): 389-402.

5. Lin TM, Beal MF. Mitochondrial dysfunction and oxidative stress in neurodegenerative diseases. Nature. 2006; 443(7113): 787-795.

6. Beal MF. Mitochondrial dysfunction and oxidative damage in Alzheimer's and Parkinson's diseases and coenzyme Q10 as a potential treatment. J Bioenerg Biomembr. 2004; 36(4): 381-386.

7. Jin H, Kanthasamy A, Ghosh A, Anantharam V, Kalyanaraman B, Kanthasamy AG. Mitochondria-targeted antioxidants for treatment of Parkinson's disease: Preclinical and clinical outcomes. 2014. Biochim Biophys Acta. 2014; 1842(8): 1282-1294. doi: 10.1016/j. bbadis.2013.09.007.

8. Tarnopolsky MA, Raha S. Mitochondrial myopathies: Diagnosis, exercise intolerance, and treatment options. Med Sci Sports Exerc. 2005; 37(12): 2086-2093.

9. Finichiu PG, James AM, Larsen L, Smith RAJ, Murphy MP. Mitochondrial accumulation of a lipophilic cation conjugated to an ionisable group depends on membrane potential, $\mathrm{pH}$ gradient and $\mathrm{pK}(\mathrm{a})$ : implications for the design of mitochondrial probes and therapies. J Bioenerg Biomembr. 2013; 45(1-2): 165-173. doi: 10.1007/s10863-012-94935 .

10. Boddapati SV, Tongcharoensirikul P, Hanson RN, D’Souza GGM, Torchilin VP, Weissig V. Mitochondriotropic Liposomes. J Liposome Res. 2005; 15(1-2): 49-58.

11. Biswas S, Dodwadkar NS, Deshpande PP, Torchilin VP. Liposomes loaded with paclitaxel and modified with novel triphenylphosphoniumPEG-PE conjugate possess low toxicity, target mitochondria and demonstrate enhanced antitumor effects in vitro and in vivo. J Control Release. 2012; 159(3): 393-402. doi: 10.1016/j.jconrel.2012.01.009. 
12. Benein P. Almuteri MA, Mehanna AS, D'Souza GG. Synthesis of triphenylphosphonium phospholipid conjugates for the preparation of mitochondriotropic liposomes. Methods Mol Biol. 2015; 1265: 5157. doi: 10.1007/978-1-4939-2288-8_4.

13. Smith RAJ and Murphy MP. Mitochondria-targeted antioxidants as therapies. Discov Med. 2011; 11(57): 106-114.

14. Armstrong JS. Mitochondrial Medicine: Pharmacological targeting of mitochondria in disease. Br J Pharmacol. 2007; 151(8): 1154-1165. doi: $10.1038 /$ sj.bjp.0707288.

15. Chinnery PF. Mitochondrial disorders overview. In Pagon RA, Adam MP, Ardinger HH, Bird TD, Dolan CR, Fong CT, Smith RJ, Stephens K, editors. GeneReviews (®). Seattle (WA): University of Washington; 1993-2015.

16. Parikh S, Saneto R, Falk MJ, Anselm I, Cohen BH, Haas R. A Modern approach to the treatment of mitochondrial disease. Curr Treat Options Neurol. 2009; 11(6): 414-430.

17. Yamada Y, Akita H, Kogure K, Kamiya H, Harashima H. Mitochondrial drug delivery and mitochondrial disease therapy-an approach to liposome-based delivery targeted to mitochondria. Mitochondrion. 2007; 7(1-2): 63-71

18. Murphy MP. Targeting antioxidants to mitochondria by conjugation to lipophilic cations. 2008. In: Dykens JA, and Will Y, editors. DrugInduced Mitochondrial Dysfunction. NJ: John Wiley \& Sons Inc; 2002. p. 577-581.

19. Murphy MP. Targeting lipophilic cations to mitochondria. Biochim Biophys Acta. 2008; 1777(7-8): 1028-1031. doi: 10.1016/j. bbabio.2008.03.029.

20. Lucken-Ardjomande S, Martinou JC. Newcomers in the process of mitochondrial permeabilization. J Cell Sci. 2005; 118(3): 473-483.

21. van der Luit AH, Budde M, Ruurs P, Verheij M, va Blitterswijk WJ. Alkyllysophospholipid accumulates in lipid rafts and induces apoptosis via raft-dependent endocytosis and inhibition of phosphatidylcholine synthesis. J Biol Chem. 2002; 277(42): 39541- 39547.

22. Marco C, Rios-Marco P, Jimenez-Lopez JM, Segovia JL, Carrasco MP. Antitumoral alkylphospholipids alter cell lipid metabolism. Anticancer Agents in Med Chem. 2014; 14(4): 545-558.

23. Fuller N, Rand RP. Influence of lysolipids on the spontaneous curvature and bending elasticity of phospholipid membranes. Biophys J. 2001; 81(1): 243-254. doi: 10.1016/S0006-3495(01)75695-0.

24.van Zutphen $H$, van Deenen LLM. The effect of lysolecithin on the electrical resistance of lecithin bilayer membrane. Chem Phy Lipids. 1967(1): 389-391. doi:10.1016/0009-3084(67)90017-5.

25. Lee Y, Chan SI. Effect of lysolecithin on the structure and permeability of lecithin bilayer vesicles. Biochemistry. 1977; 16(7): 1303-1309.

26. Horobin RW, Trapp S, Weissig V. Mitochondriotropics: a review of their mode of action, and their applications for drugs and DNA delivery to mammalian mitochondria. J Control Release. 2007. 121(3): 125-136.

27. Ross MF, Kelso GF, Blaikie FH, James AM, Cocheme, HM, Filipovska A, et al. Lipophilic triphenylphosphonium cations as tools in mitochondria bioenergetics and free radical biology. Biochemestry (Mosc). 2005; $70(2): 222-230$ 